\title{
Quadratus Lumborum Block for Robotic Abdominal Surgery: A Case Report
}

\author{
Instituto Nacional do Câncer - INCA
}

P. Milanez, MD, D. Brandão, MD, F. Robalinho, MD, D. Minelli, MD, P. Pimentel, MD, S. Lemos, PhD

\section{Introduction}

Since Enhanced Recovery Surgery (ERAS) Protocol has been implemented, there has been a growing interest in multimodal anesthesia. Regional anesthesia could be a key for this purpose. After the introduction of ultrasound guided blocks, some alternatives seem promising: Quadratus Lumborum (QL) block may be one of them. ${ }^{1,2}$

\section{Case Report}

The case was a 61-year-old woman who underwent a Robotic Hartmann Surgery. General anesthesia was achieved with lidocaine, dexmedetomidine, magnesium sulfate, rocuronium, propofol, sevoflurane and ketamine. An incision in the abdominal middle line was made, so an upgrade in analgesia was necessary. A bilateral posterior QL block was performed, at the end of surgery, as follows: the patient was in the supine position, a high-frequency transducer was placed in a transversal manner and the border between the Quadratus Lumborum Muscle (QLM) and The Latissimus Dorsi Muscle was visualized. Using a 100 millimeters needle (Stimuplex®, Ultra 22 G, Germany), 40 milliliters of $0,3 \%$ ropivacaine were injected. Additional, analgesia was: dipyrone and Tenoxicam. There was no need for opioid in the control of pain, during the postoperative period.

\section{Discussion}

The promise of more extensive abdominal analgesia compared with the Transverse Abdominal Plane (TAP) block accounts for the growing interest in QL block. This muscle along with the thoracolumbar fascia could be an anatomical bridge between the anterolateral musculature of the abdominal wall and the lumbar paravertebral region. ${ }^{2}$ The lack of consensus on the mechanism of spread for QL block may be partially attributed to the variable descriptions of the approaches: QL1 would be named lateral QL block; the QL2 block would be called posterior QL block, and the transmuscular QL block would be an anterior QL block. ${ }^{3}$ The choice of analgesia in the operating room and postoperatively will have an impact on elements of the ERAS protocol and minimizing opiates by using regional blocks could be one of the keys. ${ }^{1}$

\section{Learning point}

There is a lack of data about QL block with Robotic surgery and this report was a successful case.

\section{Acknowledgment}

Written informed consent was obtained

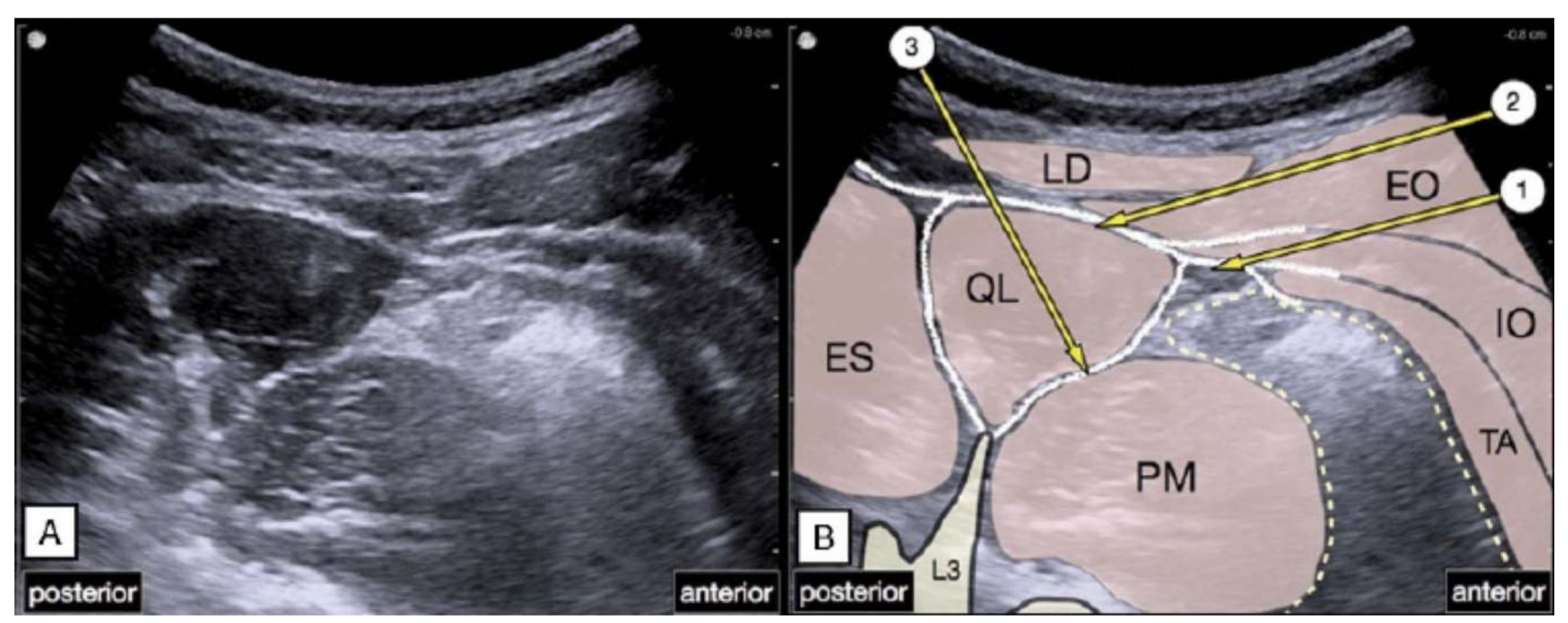

Figure 1: Essentials of Our Current Understanding: Abdominal Wall Blocks (Chin J. K. , 2017)

1- Feldman S. L., Anesthesia and Analgesia Protocol. Current Surgical Therapy. 2017, 325-334.

2- Chin J. K., Essentials of Our Current Understanding: Abdominal Wall Blocks. Reg Anesth Pain Med. 2017; 42:133-183.

3- Elshakawy H., Quadratus Lumborum Blocks. Advances in Anesthesia. 2017; 35: 145-157. 